\title{
Médecine, Science et Génétique Les années charnières
}

Comment pouvons-nous le mieux résumer l'année 1994, vue de médecine/sciences? D'un point de vue scientifique et médical - et nous tomberons là-dessus d'accord avec la revue Science - la grande avancée est certainement la promotion des systèmes de réparation de l'ADN au rang de vedettes. Jusqu'en 1993, ces systèmes n'étaient réellement considérés que par quelques spécialistes de l'ADN et biologistes théoriciens qui savaient combien la réparation des erreurs est essentielle à la stabilité de l'information génétique, et par quelques médecins s'occupant de ces affections un peu ésotériques réunies sous le nom de "réparatoses" (ataxie-télangiectasie, maladie de Fanconi, syndrome de Bloom, xeroderma pigmentosum... dans lesquelles des anomalies de la réparation de l'ADN avaient été suggérées, puis démontrées). On savait cependant que la labilité génétique des cellules cancéreuses, facteur de progression tumorale et de résistance aux traitements, était une des caractéristiques du cancer expliquant en grande partie les difficultés rencontrées dans la mise au point d'un traitement radical de ces affections. En 1993, fut localisé un gène de susceptibilité au cancer du côlon associé à une instabilité généralisée de séquences répétées du génome appelées "microsatellites" $\left(\mathrm{m} / \mathrm{s} \quad n^{\circ} 8 / 9\right.$, vol. 9, p. 900). Puis, fin 1993, ce fut le clonage du gène $h M S H 2$, équivalent humain des gènes de $E$. coli et de levure intervenant dans la réparation des mésappariements $\left(\mathrm{m} / \mathrm{s} \quad n^{\circ} 2\right.$, vol. $10, p .228$ ). Le premier gène devait être rapidement suivi de plusieurs autres, $h M L H 1$, $h P M S 1$ et $h P M S 2$, formant ensemble les gènes responsables du "syndrome de Lynch ", forme de cancer colique familial sans polypose, parfois associé à d'autres lésions $\left(\mathrm{m} / \mathrm{s} n^{\circ} 1\right.$, vol. 10 , p. 1178); tous ces gènes interviennent dans le même processus de ré- paration de l'ADN que hMSH2. Ce mécanisme de susceptibilité génétique au cancer - la perte de l'efficacité des systèmes de réparation facilitant l'accumulation de mutations dont certaines doivent être tumorigènes - n'est probablement pas limité au cancer du côlon, car ce phénotype "mutateur" avec instabilité des microsatellites est aujourd'hui retrouvé dans une grande diversité de cancers. Il faut observer que si la découverte des gènes de susceptibilité du type hMSH2, MLH1 est bien essentielle sur le plan de la compréhension des mécanismes de la cancérogenèse et du développement des tests génétiques de prédisposition, elle n'ouvre guère de nouveaux espoirs thérapeutiques, au moins à court terme. La même remarque pourrait d'ailleurs être faite pour les affections, en nombre croissant, caractérisées par une expansion de triplets nucléotidiques, maladies dominantes (à l'exception du syndrome de l'X fragile) à manif estations neurologiques (à l'exception de la dystrophie myotonique de Steinert) $\left(\mathrm{m} / \mathrm{s} n^{\circ} 4\right.$, vol. $\left.10, p .472\right)$. Telle est l'une des principales caractéristiques de l'évolution de la médecine à l'heure de l'explosion des études sur le génome: le mécanisme des maladies sera de mieux en mieux connu, les possibilités de les prévoir avant l'apparition de tout symptôme se multiplieront, mais, pour un temps, les moyens manqueront souvent pour passer de la prédiction à la prévention et au traitement. Il n'y a pas de doute que cette situation du médecin "voyeur impuissant", situation temporaire, espérons-le, marquera cette fin de siècle et modifiera les rapports des praticiens avec leurs malades.

Toujours sur le plan de l'avancée des connaissances, on peut relever, en 1994, le nombre croissant d'affections liées à des mutations activatrices ou inactivatrices des récep- teurs membranaires à sept passages transmembranaires (par exemple, la maladie de Hirschsprung et la susceptibilité aux endocrinopathies néoplasiques multiples de type 2: $m / s n^{\circ} 4$, vol. $10, p .450$; l'achondroplasie: $m / s n^{\circ} 8-9$, vol. $10, p .936$; la craniodysostose de Crouzon: $\mathrm{m} / \mathrm{s}$ $n^{\circ} 11$, vol. 10, p. 1163).

Une autre avancée essentielle, prolongeant à vrai dire une tendance antérieure à l'année 1994, est l'articulation croissante démontrée entre les différents systèmes réglant la vie et l'activité cellulaires : le facteur de transcription BTF2/TFIIH comporte des sous-unités qui sont impliquées dans la réparation et correspondent à des gènes de réparatose: $\mathrm{m} / \mathrm{s} n^{\circ} 10$, vol. $\left.10, p .973\right)$; le cycle cellulaire, la réplication, l'apoptose, la réparation, la sénescence sont interconnectés par un jeu de kinases, d'inhibiteurs de kinase, de facteurs de transcription... et de p53 $(\mathrm{m} / \mathrm{s}$ $n^{\circ} 2$, vol. 10, p. 206; $n^{\circ} 6-7$, vol. 10, p. 744).

Toutes ces découvertes... et bien d'autres, aboutissent à la création de familles de "candidats" au titre de gènes de susceptibilité à diverses maladies génétiques, ou de gènes impliqués dans des situations physiophathologiques particulières, et donc, potentiellement, à l'identification de cibles thérapeutiques. En effet, l'observation qu'un type de gène est responsable, par ses anomalies d'une maladie amène à se demander si des gènes voisins ne pourraient pas, eux aussi, expliquer d'autres affections plus ou moins proches. C'est ainsi que les gènes $h M L H 1, h P M S 1$ et $h P M S 2$ ont été découverts sur la base de leur ressemblance à des gènes de réparation de l'ADN connus chez le colibacille et la levure. Actuellement, de nombreuses équipes tentent d'impliquer des gènes codant pour des facteurs de réparation, des récepteurs membranaires et bien d'autres familles 
de protéines dans le mécanisme de nombreux désordres, génétiques ou non. Pour qu'une telle recherche soit efficace, il faut avoir accès à la plus grande collection possible de séquences de gènes... ou d'ADNc humains. Une telle collection existe; elle est le fruit des efforts d'un consortium privé, financé avant tout par la compagnie SmithKline Beecham (SKF) : Human Genome Science (HGS) et sa fondation TIGR ont séquencé 380000 fragments d'ADN complémentaires tirés de 380 librairies dans E. coli, leur permettant d'identifier les séquences partielles entre 40000 et plus de 50000 gènes parmi les 70000 à 100000 gènes probablement exprimés chez les humains. Un effort gigantesque est consenti maintenant pour positionner ces séquences sur les cartes génétiques et physiques du génome. Quand cela sera réalisé - dans quelques années - la découverte des gènes de maladie deviendra simple: les gènes candidats suggérés par la physiopathologie ou la localisation génétique seront disponibles et leur responsabilité pourra être rapidement testée.
Tout est fort bien, par conséquent... sauf que l'accès à cet outil irremplaçable n'est pas gratuit, qu'il est même fort contraignant, HGS/SKF réclamant le "droit de premier refus", c'est-à-dire une priorité pour développer toutes les recherches lui semblant économiquement intéressantes. Cet épisode est assez emblématique du paysage de la recherche en génétique et biotechnologie tel qu'il s'est dessiné nettement cette année. Le remarquable succès des firmes privées $\mathrm{HGS} / \mathrm{SKF}$ renouvelle le genre inauguré par le Généthon financé massivement par l'Association Française de lutte contre les Myopathies, celui d'une progression en dehors des circuits de la recherche académique. De plus, l'investissement est, ici, un capital dont la raison d'être est d'engendrer un retour sur investissements, d'où les règles édictées par HGS/SKF pour utiliser leurs données.

Ce qui est fantastique, dans l'évolution de la médecine et de la science biologique aujourd'hui, c'est qu'elle n'est pas seulement prodigieuse quant à sa rapidité et à la largeur du panorama auquel elle donne accès sur les mécanismes de la vie et leurs pertubations, mais aussi qu'elle est un reflet, voire une caisse de résonance, des tendances fortes de nos sociétés dans les domaines de l'économie et de la morale. De tous ces aspects, $m / s$ s'est voulu un témoin privilégié. Votre confiance croissante nous laisse penser que nous nous sommes correctement acquittés de cette tâche de témoignage, ce qui nous donne tout le courage nécessaire pour entrer avec détermination dans notre... $11^{\mathrm{e}}$ année: que d'événements nous aurons à commenter et à fêter en 1995 !... à commencer par notre dixième anniversaire auquel nous espérons que vous serez nombreux à participer, le 16 mars prochain à Paris et le 27 mars à Montréal.

Bonne année à tous nos lecteurs, bonne année à notre $\mathrm{m} / \mathrm{s}$, que l'année 1995 soit féconde sur le plan des connaissances et riche sur celui de la qualité de nos réflexions sur leurs conséquences pour la personne et la société... une bonne année de $m$ decine/sciences.

\section{Michel Bergeron}

Axel Kahn

$R$ dacteur en chef

$R$ dacteur en chef

Montréal

Paris

Les Editions John Libbey Eurotext Gilles Cahn et ses collaborateurs vous présentent leurs meilleurs vœux pour 1995 Les Editions John Libbey Eurotext Gilles Cahn et ses collaborateurs vous présentent leurs meilleurs $\sqrt{\text { John Libbey }}$ Les Editions John Libbey Eurotext Gilles Cahn et ses collaborateurs vous tions John Libbey Eurotext Gilles Cahn et ses collaborateurs vous présentent leurs meilleurs vœux pour 1995 Les Editionam Libbey Euroten présentent lo et ses co Libbey Eurot 1995 Les $\mathrm{H}$ tent leurs me collaborateur: Eurotext Gill Les Editions

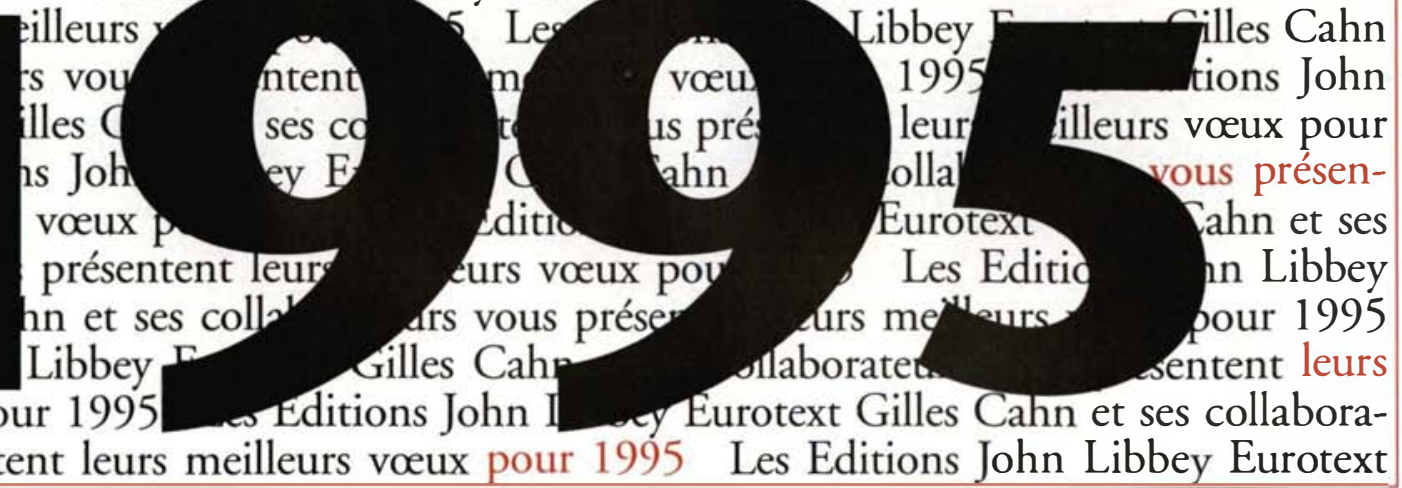
meilleurs vœux pour 1995 s Editions John I ey Eurotext Gilles Cahn et ses collaborateurs vous présentent leurs meilleurs vœux pour 1995 Les Editions John Libbey Eurotext 